
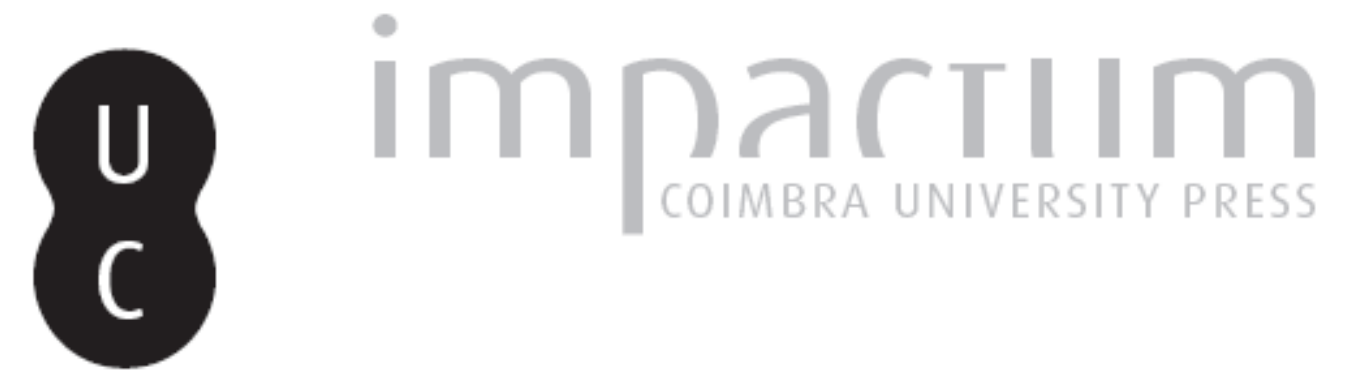

\title{
La philosophie du mariage chez Plutarque
}

Autor(es): Boulogne, Jacques

Publicado por: International Plutarch Society

URL persistente:

URl:http://hdl.handle.net/10316.2/36374

DOI:

DOI:http://dx.doi.org/10.14195/0258-655X_7_2

Accessed : $\quad$ 26-Apr-2023 12:28:34

A navegação consulta e descarregamento dos títulos inseridos nas Bibliotecas Digitais UC Digitalis, UC Pombalina e UC Impactum, pressupõem a aceitação plena e sem reservas dos Termos e Condições de Uso destas Bibliotecas Digitais, disponíveis em https://digitalis.uc.pt/pt-pt/termos.

Conforme exposto nos referidos Termos e Condições de Uso, o descarregamento de títulos de acesso restrito requer uma licença válida de autorização devendo o utilizador aceder ao(s) documento(s) a partir de um endereço de IP da instituição detentora da supramencionada licença.

Ao utilizador é apenas permitido o descarregamento para uso pessoal, pelo que o emprego do(s) título(s) descarregado(s) para outro fim, designadamente comercial, carece de autorização do respetivo autor ou editor da obra.

Na medida em que todas as obras da UC Digitalis se encontram protegidas pelo Código do Direito de Autor e Direitos Conexos e demais legislação aplicável, toda a cópia, parcial ou total, deste documento, nos casos em que é legalmente admitida, deverá conter ou fazer-se acompanhar por este aviso.

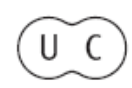




\section{PLOUTARCHOS, n.s.}

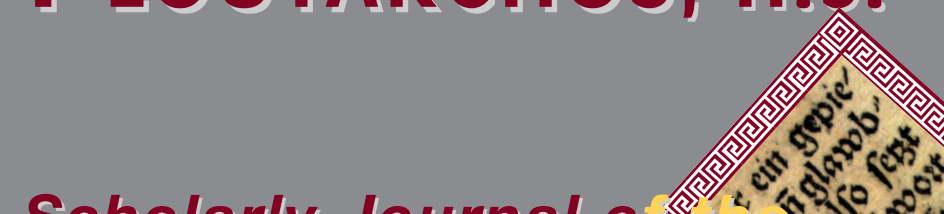

\section{Scholarly Journal o}

International Plut
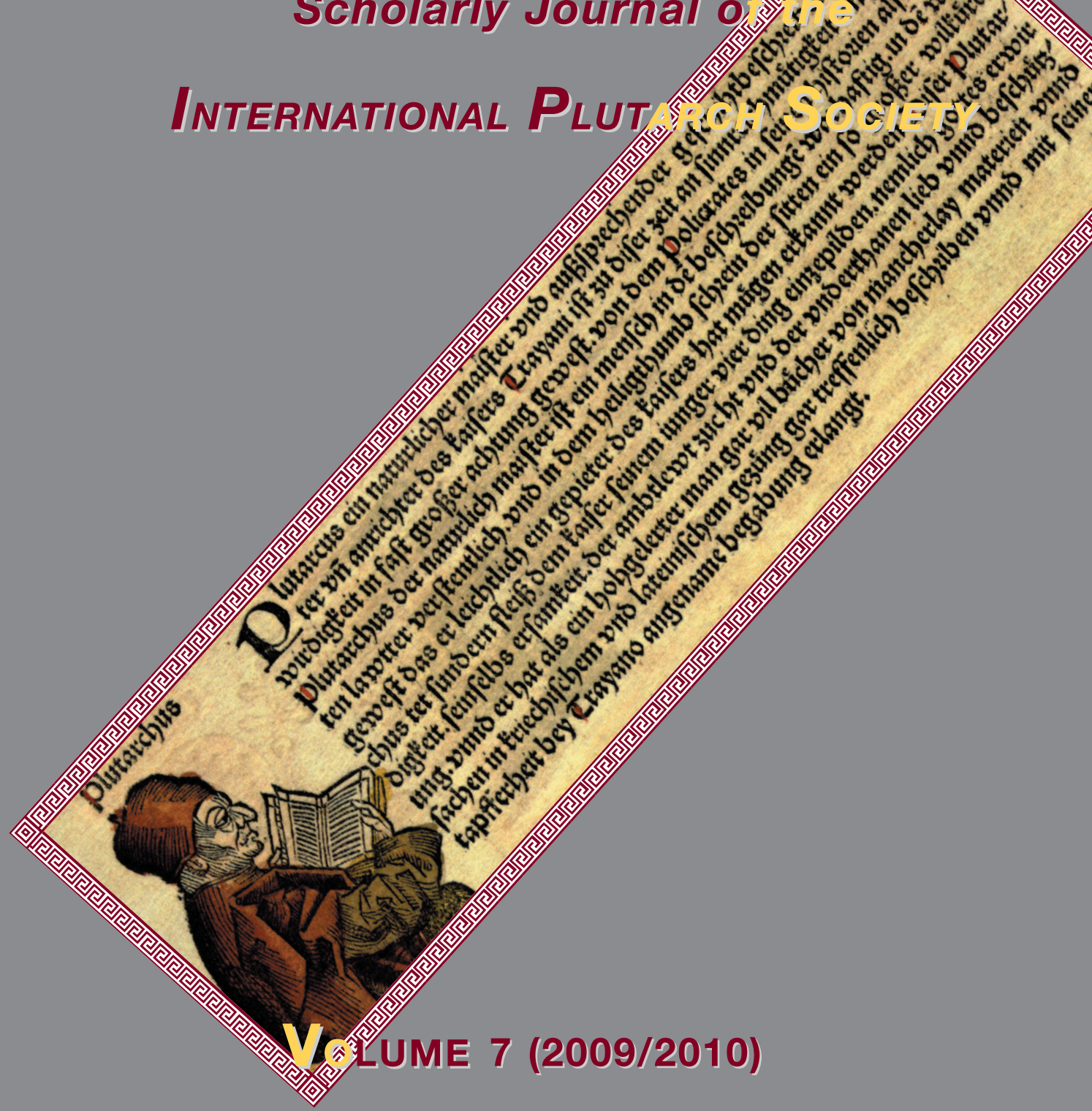

UNIVERSITY OF MÁLAGA (SPAIN) Utah State University, Logan, Utah (U.S.A.) 


\title{
La philosophie du mariage chez Plutarque par Jacques Boulogne
}

\section{Université Charles de Gaulle-Lille 3, HALMA-IPEL, CNRS/UMR 8142} jacques.boulogne@univ-lille3.fr

\begin{abstract}
In Plutarch's writings, we find a very original conception of marriage. Much more than a harmonious living together, full success concerning marriage, as regards physical pleasure, sentiments, and intellectual connivance, looks like a real symbiosis, the paradigm of which is provided by physics through the integral mixing, namely crasis. Carrying out such an union of male and female principles, in Plutarch's opinion, requires from the wife a philosophical being in sympathy with her husband's views about the world and, to Plutarch's mind, reaches nearly a mystic level.

Key-Words: Plutarch, Quaestiones romanae, Conjugalia praecepta, Amatorius, De Iside et Osiride, Hesiod, Homer, Plato, Aristotle, Xenophon, Chrysippos, Philo of Alexandria, Confidence, Fidelity, Friendship, Esteem, Integral mixing, Crasis, Christian values, Nuptial number.
\end{abstract}

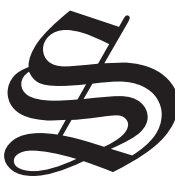

ujet qui concerne aussi bien l'homme de la rue, préoccupé de son bonheur personnel, que les dirigeants, qui ont en charge les institutions de la société, le mariage ne pouvait pas ne pas intéresser Plutarque. De fait, beaucoup a déjà été écrit sur les femmes et le mariage dans l'œuvre de Plutarque. Nous pensons en particulier à la très substantielle étude d'ensemble effectuée par Nikolaidis sous le titre " Plutarch on Women and Marriage » où est affirmée l'influence sur la pensée de ce Grec de Chéronée de la position sociale de la matrone romaine, sensiblement supérieure à celle de son homologue athénienne ${ }^{1}$. Notre propos n'est pas de contester l'admiration qu'éprouve cet auteur pour nombre de coutumes observées à Rome, notamment au sujet des femmes, comme en témoigne ne serait-ce que sa totale approbation de l'extension aux épouses de l'éloge funèbre, alors qu'en Grèce la bienséance veut qu'on parle d'elles le

1 Nikolaidis (1997), 28. 
moins possible ${ }^{2}$. Toutefois il semblerait que ses conceptions en la matière doivent plus à ses idées philosophiques qu'à l'observation du couple romain et même que ces dernières aient orienté sa lecture des mœurs conjugales pratiquées par les Romains. Il déclare en effet, dans la préface de son traité Préceptes de mariage, qu'il n'existe pas de thème plus philosophique que celui du mariage (138 B). Il s'agit là d'une question sur laquelle il réfléchit depuis le début de son propre mariage, si l'on en croit la confidence autobiographique qu'il nous livre au cours du prologue de son Dialogue sur Éros (2, 749 B), et il apparait plus que probable que les problèmes de sens soulevés à ses yeux par tel ou tel rite en vigueur à Rome reçoivent leur élucidation dans les Étiologies romaines de ce qu'il serait légitime d'appeler sa philosophie du mariage. Or cette philosophie n'est qu'effleurée par Patterson, qui, il est vrai, centre son analyse sur les Préceptes de mariage ${ }^{3}$ : bien qu'elle reconnaisse que Plutarque aborde la relation matrimoniale à travers une loupe philosophique, elle montre que l'intérêt de l'essai ne réside pas dans l'originalité, ni dans le reflet d'un climat moral nouveau ${ }^{4}$, mais dans le mélange de considérations empruntées à des écoles philosophiques différentes et à la tradition populaire, afin de rassembler, un peu comme le fait, en 1824-1829, Balzac dans son ouvrage Physiologie du mariage, ou méditations de philosophie éclectique sur le bonheur et le malheur conjugal, tous les conseils censés aider à éviter l'échec ${ }^{5}$. Nous n'allons pas reprendre à notre tour l'étude de ce texte ${ }^{6}$ "nous n'apporterions rien de bien nouveau par rapport à tout ce qui a pu être dit au long des deux dernières décennies", mais plutôt essayer de compléter en nous appuyant sur d'autres écrits de Plutarque. À cette fin, nous passerons en revue les valeurs associées au mariage au point de former un véritable système axiologique, puis nous verrons sur quel modèle physique cette axiologie est pensée, avant d'en dégager pour finir les postulats métaphysiques.

\section{Une axiologie de l'harmonie}

La préface des Préceptes de mariage setermine surl'idéed'harmonie présentée comme la clé du bonheur conjugal (138 C). Afin de mieux faire comprendre ce qu'il veut dire, Plutarque recourt à

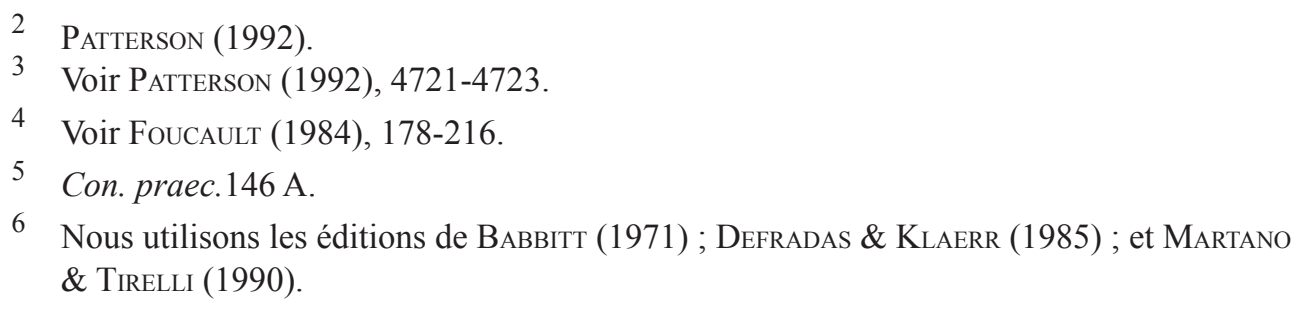


l'image de la lyre dont les cordes bien accordées vibrent, non pas à l'unisson car alors la polyphonie disparaîtrait au profit d'un son unique, ce qui constituerait un appauvrissement, mais d'une manière consonante ( $\dot{\varepsilon} \mu \mu \dot{\lambda} \lambda \varepsilon 1 \alpha v)$. Nous avons affaire à l'oxymore d'une union qui n'est pas réductrice à l'un, mais où l'un reste double et où la dualité constitue une unité. Et il ajoute qu'un tel résultat ne peut s'obtenir qu'au prix du concours de plusieurs divinités : Aphrodite, les Muses, Hermès, Peïtho et les Kharites. On pourrait s'étonner de l'absence d'Éros. Mais, à la différence de l'Érotikos où Plutarque célèbre la mariage d'amour parce que le thème principal est précisément l'amour, ici le sujet demeure le mariage, qui, grâce aux Muses, dépasse l'amour : comme nous le verrons, Éros laisse la place à la Muse Érato.

La première est associée au plaisir physique dont l'existence ne doit pas être réprimée même si son importance demeure minime ${ }^{7}$, tandis que la troisième représente plutôt la parole rationnelle de la communication ${ }^{8}$. Peïtho et les Kharites ${ }^{9}$ correspondent à un état d'esprit caractérisé par la volonté de gagner la confiance au moyen de la persuasion et de la complaisance mutuelle. Quant aux Muses, en tant que filles de Mémoire et gardiennes de la culture commune ${ }^{10}$, elles harmonisent de leur musique les différents secteurs du monde sublunaire, notamment en présidant aux diverses manifestations du désir de plaisir, qui reste la motivation dernière de toute activité ou action. C'est ainsi que dans les Propos de Table ${ }^{11}$, Érato a pour fonction de civiliser l'ardeur amoureuse en lui retirant ce qu'elle comporte de violent et de débridé de manière à ce qu'elle débouche sur l'amitié ( $\varphi \imath \lambda i ́ \alpha v)$ et

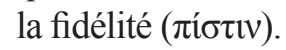

Comme nous le constatons, Plutarque reprend partiellement le mythe de la création de Pandore parée en vue de son mariage avec Épiméthée par Aphrodite, les Khrarites, Peïtho et Hermès ${ }^{12}$. Mais surtout il corrige la version d'Hésiode : chez lui, Hermès cesse d'être l'inspirateur du mensonge,

7 En particulier, le commerce sexuel est recommandé comme la meilleure des médecines pour régler les différends entre époux (Con. praec. 143 D ; cf. 138 D et Amatorius 769 C-D). Pour la réhabilitation du corps en général par Plutarque, voir son traité sur l'hygiène de vie, De tuenda sanitate praecepta.

8 Voir la monographie de KAHN (1978). Voir BenVÉniste (1969), 103-121.

9 Sur leur symbolisme, voir l'interprétation de Sénèque, Benef. 1, 3.

10 Con. praec. $146 \mathrm{~A}$.

11 Quaest. conv. 746 E-F (= livre 9, Problème 15 « Traditions rares sur le nombre des Muses »).

12 Voir Hésiode, $O p .65-82$. 
de la tromperie et de la dissimulation, pour patronner le raisonnement philosophique épris de vérité et qui cherche le bien de l'autre. Une fois que le manque propre au sentiment amoureux se trouve comblé grâce à la réciprocité de l'amour partagé, la passion se transmute en amitié, dont la nature porte précisément à vouloir l'épanouissement de l'être aimé $^{13}$. Et cette amitié s'accompagne nécessairement de fidélité, puisqu'elle repose sur le partage de la volonté de complaire et donc de répondre aux attentes. L'échange de la générosité crée la confiance, laquelle exclut l'infidélité, car elle signifie remettre son sort au pouvoir d'une puissance dont on souhaite recevoir la protection ${ }^{14}$.

Le don de soi qu'implique la confiance et qui, sous la tutelle des Kharites, se traduit entre autres par la grâce, requiert également le respect mutuel. C'est quand on se sent estimé qu'on se livre sans défense, parce qu'alors on sait qu'on n'a rien à craindre. Voilà pourquoi
Plutarque écrit : « Et si la part du plaisir est peu de chose, l'estime qui en germe chaque jour, le don de soi, l'affection réciproque et la confiance ne réfutent de sottises ni chez les Delphiens quand ils appellent Aphrodite Arma (i-e Union), ni chez Homère qui donne à l'intimité de ce genre le nom de tendre amitié $\gg{ }^{15}$. Les voluptés physiques symbolisées par Aphrodite ne sont donc pas condamnées. Elles ne deviennent pas pour autant une fin en soi. Le rôle de cette déesse, est-il rappelé dans le Banquet des Sept Sages $(13,156 \mathrm{C})$, ne se borne pas à favoriser la sexualité : les jouissances que celle-ci engendre ont en réalité pour conséquence de faire fondre ensemble les âmes et de les mêler étroitement aux corps afin de produire « l'accord des pensées et l'amitié $\gg{ }^{16}$.

Il apparaît ainsi que les valeurs ouvrières de la solidité du mariage se ramènent à trois : l'estime, le don de soi, la confiance. Toutes les autres valeurs, fidélité, pudeur ${ }^{17}$, solidarité,

13 Voir Benvéniste (1969), 335-353 : il montre que le mot pí̉o et ses dérivés désignent fondamentalement l'accueil généreux et confiant réservé à un étranger. Voir aussi Platon, Ly., en particulier 207 d-e.

14 Voir Benvéniste (1969), 103-121.

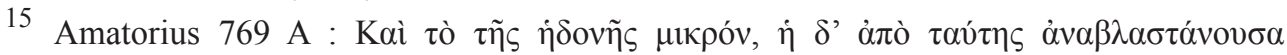

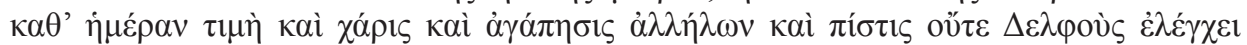

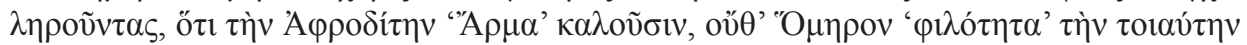

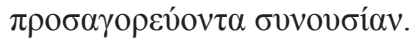

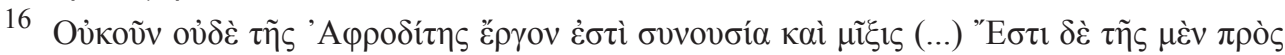

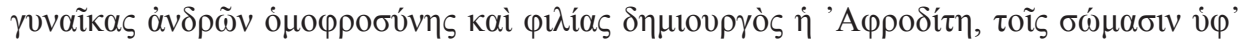

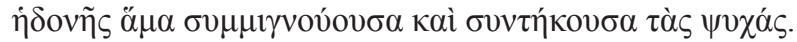

17 Pour ce sentiment de déférence qu'est l' aỉós, voir Con. praec. 139 C et 144 F - 145 A, ainsi que BENVÉNISTE (1969), 340-341. 
entente, amitié, persuasion, plaisirs sexuels, leur demeurent subordonnées. Elles sous-tendent l'ensemble des recommandations dispensées dans les Préceptes de mariage. Elles se retrouvent aussi dans l'interprétation que propose Plutarque des rites conjugaux à Rome. Par exemple, lorsqu'il essaie de comprendre pourquoi les Romains allument cinq cierges lors des cérémonies nuptiales, il suggère que leurs croyances réclament autour des jeunes mariés la présence de cinq divinités protectrices, dont Aphrodite et Peïtho ${ }^{18}$. Ou encore, s'agissant de l'interdiction d'accepter entre époux des dons mutuels, il préfère l'explication d'après laquelle le mariage rend absolument communs tous les biens des conjoints ${ }^{19}$.

Précisons encore que les valeurs qui président à la réussite du mariage, surtout la grâce et la communion des cœurs et des esprits, se rencontrent déjà chez Homère, qui, au chant 14 de l'Iliade, développe une méditation sur le mariage et ses échecs en laissant entrevoir un modèle de réussite avec le futur couple formé par Hypnos et Pasithéa, précisément une Kharite (267276) ; ajoutons que, dans l'Odyssée (VI, 181-183), il fait souhaiter par Ulysse à Nausicaa qui rêve de mariage de connaître une union marquée par

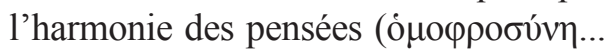

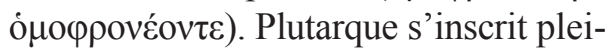

nement dans le droit fil de la tradition homérique.

\section{Le paradigme du mélange intégral}

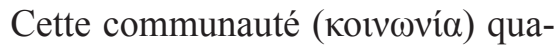
si fusionnelle du couple est pensée sur le modèle du mélange. Mais pas de n'importe quel mélange. Les Grecs distinguent en effet plusieurs types de mélange $(\mu \tilde{i} \xi 1 \zeta)$. Plutarque nous en établit une rapide récapitulation dans le chapitre 34 des Préceptes de mariage (142 E - 143 A). Selon un ordre croissant dans l'unification, il mentionne d'abord les ensembles constitués par la réunion d'éléments qui restent séparés, tels par exemple les navires d'une même flotte. Puis, passant du rassemblement à l'assemblage, il évoque les mélanges composés de pièces jointes ensemble par ajustement, comme dans le cas d'une maison ou d'un navire. Enfin, au-dessus de la contiguïté en matière de cohésion, il situe la coalescence

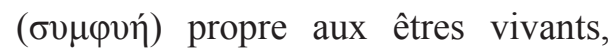
dont les parties ont poussé ensemble, soudées les unes aux autres sans qu'il y ait de joints apparents.

Appliquant ensuite cette classification à l'union conjugale, il dresse une brève typologie du mariage en fonction de sa motivation. Si celle-ci se cantonne au seul plaisir charnel, nous avons affaire au côtoiement de deux époux qui dorment dans le même lit

19 Quaest. Rom. 265 F - 266 A, cf. Con. praec. 152 D-E et 143 A. 


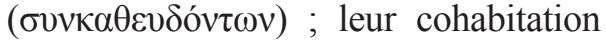

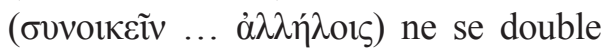

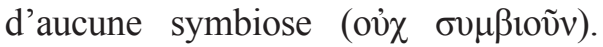
Il n'y a pas non plus partage de la vie dans le cas des couples dont les biens se limitent à des considérations uniquement matérielles (dot, patrimoine pour lequel il faut donner une postérité). Seuls les mariés unis par l'amour atteignent à l'unité, sans solution de continuité ou marque de séparation, de deux existences fondues en une seule. Afin de renforcer cette idée de force cohésive maximum, Plutarque ajoute à la métaphore de l'unité de l'organisme vivant une comparaison empruntée à la physique des fluides avec le mélange des liquides qui s'interpénètrent intégralement $(\delta \mathrm{t}$ ö $\lambda \omega v$... $\kappa \rho \tilde{\alpha} \sigma \mathrm{\sigma})$ au point de donner naissance à une substance parfaitement homogène, dont toutes les parties sont semblables entre elles, autrement dit une substance homéomère à l'instar du vin coupé d'eau $(\kappa \rho \tilde{\alpha} \mu \alpha)^{20}$. L'expression est

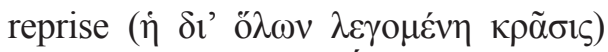
dans le Dialogue sur Éros (24, 769 F), où les époux qui vivent sous le même toit sans amour sont comparés à un agrégat d'atomes qui s'accrochent et s'entrechoquent continuellement avant de finir par provoquer une désagrégation.

L'image, par conséquent, la plus pertinente pour représenter le mariage heureux se révèle être la crase, autrement dit celle d'une fusion sans confusion ( $\sigma u ́ v \chi v \sigma i \varsigma)$. De même que le mélange intégral ne signifie pas synthèse et altération des composants provoquant l'apparition d'un produit essentiellement différent, et qu'il est possible par décomposition de retrouver les ingrédients intacts, comme on peut séparer l'eau du vin ${ }^{21}$ ou le sel de l'eau, de même la symbiose du mariage d'amour ${ }^{22}$ ne se réalise nullement au prix du renoncement de l'un ou l'autre conjoint à l'épanouissement de son être. Bien loin que l'un doive se sacrifier pour le bonheur de l'autre, ils s'aident l'un l'autre à aller jusqu'au bout de lui-même : au lieu d'avoir affaire à une dénaturation, nous assistons plutôt à un parachèvement.

Comme l'analyse parfaitement Babut dans sa thèse ${ }^{23}$, si des recoupements avec le stoïcisme et plus précisément avec le stoïcisme récent ${ }^{24}$ apparaissent

20 Con. praec. 140 F. En grec moderne, le vin se dit кpá

21 Avec une éponge huilée. Voir Philo, Conf. 184 (= v. Arnim, SVF II, 472 (p. 153).

22 Voir le fragment 204 (SANDBACH).

23 BABUt (1969), 109-110.

24 Antipater de Tarse, un défenseur lui aussi du mariage comme Musonius Rufus et Hiéroclès, oppose l'affection existant entre époux aux autres affections, qui lui paraissent moins complètes et par là même moins véritables, parce qu'elle signifie communauté non seulement des biens, des amis et des enfants, mais également des corps et des âmes ; et, 
difficilement évitables, les perspectives demeurent trop différentes pour autoriser à parler d'influence exclusive. Certes les Stoïciens contribuent au développement de la réflexion sur les mélanges en distinguant ${ }^{25}$ la juxtaposition $(\pi \alpha \rho \alpha \dot{\theta} \theta \varepsilon \sigma 1 \varsigma)$ caractérisée par un simple contact des surfaces des corps réunis, de la mixtion ( $\mu \tilde{i} \xi 1 \zeta$ ) caractérisée, elle, par la coextension

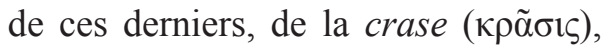
qui ne diffère de la mixtion que par sa nature liquide, et de la confusion ( $\sigma 0 ́ \gamma \chi v \sigma 1 \varsigma$ ), qui se traduit par une transformation qualitative des ingrédients dont la synthèse ( $\sigma 0 ́ \gamma \chi v \sigma ı)$ génère un produit totalement nouveau. Plutarque connaît bien le dossier, comme en témoigne sa polémique contre la physique de Chrysippe sur la question du déplacement d'un corps plein à l'intérieur d'un autre corps plein, une thèse dont l'affirmation entraîne pour conséquence que l'enveloppant soit simultanément l'enveloppé et vice-versa ou que, par exemple, une goutte de vin jetée dans la mer se répande sur toute l'étendue de celle-ci ${ }^{26}$. Comme le montre précisément cette polémique, si Plutarque emprunte une terminologie stoïcienne, il ne va pas jusqu'à adopter la théorie qui l'accompagne sur les éléments de la matière. De plus, les distinctions terminologiques des Stoïciens étaient plus ou moins tombées dans le domaine public et devenues, à cause de leur succès, propriété collective, si l'on en juge par l'utilisation qu'en fait également Philon d'Alexandrie ${ }^{27}$. Enfin et surtout, la notion de crase n'est pas une invention stoïcienne. Largement employée par la médecine hippocratique au sujet des humeurs et du mélange des qualités élémentaires que sont le chaud et le froid, le sec et $1^{\prime}$ humide ${ }^{28}$, elle se trouve également au centre des spéculations de Platon sur le composé que constitue pour lui l'âme du monde, dont la substance purement intelligible mêle en elle d'une manière absolument homogène le Même, l'Autre et le Mixte $^{29}$, de même que dans son explication de l'impression sonore de fondu que donne le phénomène acoustique de la consonance ( $\sigma v \mu \varphi \omega v i ́ \alpha)$, l'aigu et le grave ne formant plus ensemble qu'un son unique et double à la fois,

afin de caractériser une telle communauté, il l'assimile aux mélanges intégraux ( $\tau \alpha$ ĩ $\delta \mathrm{i}$ '

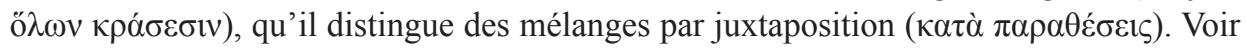
v. ARNim, SVF III, 63 (p. 255, 1. 11-18).

25 Voir v. ARNim, SVF II, 471 (p. 153).

26 De comm. not. 1077 E-1078 E. Plutarque suit, ici, Aristote (cf. GC III, 10, 318 a 26-28).

27 Voir Conf. 184 (= v. ArNim, SVF II, 472 (p. 153).

28 Voir en particulier le traité Nat. Hom., Jouanna (1975).

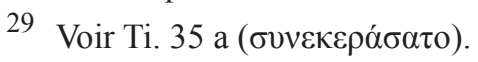


un son homogénéisé, grâce à leur crase $(\sigma v v \varepsilon \kappa \varepsilon \rho \alpha ́ \sigma \alpha \tau o)^{30}$. Elle est ensuite reprise par les Péripatéticiens dans le cadre de leurs recherches sur la mixtion $^{31}$. Mais indiscutablement, par delà les ressemblances terminologiques avec le stoïcisme, l'assise philosophique de l'application de la notion de crase à l'union conjugale idéale demeure d'inspiration platonicienne.

\section{Une religion mystérique}

En tant qu'agent d'une crase humaine, les époux reconstituent en quelque façon l'unité primordiale de l'androgyne ${ }^{32}$, ou de l'humanité non encore sexuellement différenciée d'avant la création de la première femme $^{33}$. En tout cas, ils participent " pour ainsi dire à de grands rites", écrit Plutarque dans son Dialogue sur Éros $(23,768$ F - 769 A : ஸ̋ $\approx \varepsilon \rho \rho$

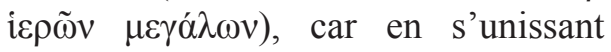
sexuellement ils renouvellent leur couple, font croître entre eux l'amitié et ainsi, à leur échelle, œuvrent à l'harmonie du monde, de même que la hiérogamie de Zeus et d'Héra, au sommet du Mont Gargare, refonde symboliquement le mariage et la cohésion cosmique, bien qu'il s'agisse d'une ruse ${ }^{34}$. C'est pourquoi Plutarque apprécie la législation de Solon qui pousse les maris à honorer leur femme le plus souvent possible ${ }^{35}$.

Bien que, par ailleurs, il compare le mariage à un labour sacré, le plus sacré de tous, car il a pour fin la procréation d'enfants ${ }^{36}$, Plutarque pense moins à la survie de l'espèce humaine qu'à la célébration de l'harmonie du monde, comme le suggèrent les deux premières des Étiologies romaines. Dans l'une (Pourquoi imposent-ils à la mariée un contact avec du feu et de l'eau ?), il rapproche l'élément masculin du couple du feu, principe de mouvement, et l'élément féminin de l'eau, puissance de la matière, pour inviter à conclure que ces opposés, bien loin de s'exclure, ont réciproquement besoin l'un de l'autre pour connaître

30 Ti. 80 b.

31 Voir Aristote, GC I, 10, 327 a $30-328$ b 22, et notamment 328 a 8-9, où la crase et la mixtion sont distinguées de la composition ( $\sigma 0 ́ v \theta \varepsilon \sigma 1 \zeta)$ dans laquelle les composants conservent leurs qualités propres, à la différence de ce que disent les Stoïciens. Voir surtout Alexandre d'Aphrodise, qui reprend la totalité du dossier dans son traité Mixt., TodD (1976).

32 Voir le mythe que Platon prête à Aristophane dans le Smp.189 d-193 d.

33 Voir le mythe de Pandore raconté par Hésiode (Th. 570-612 ; Op. 60-105).

34 Voir Il. XIV, 346-353.

35 Voir Amatorius 769 A ; cf. Con.praec. 138 D-E ; 143 C-E.

36 Con. praec. $144 \mathrm{~B}$. 
chacun sa propre plénitude, le feu se nourrissant de l'eau et l'eau recevant sa fécondation du feu ${ }^{37}$. L'institution du mariage consacre donc cette dimension cosmique de l'union sexuelle. L'explication de la seconde coutume nuptiale (Pourquoi dans les mariages allumentils, ni plus ni moins, cinq flambeaux auxquels ils donnent le nom de cierges ?) accentue l'interprétation métaphysique du mariage. Elle nous fait entrer en effet dans la symbolique des nombres en enseignant que le premier impair, le nombre trois, correspond au masculin, tandis que le premier pair, le nombre deux, correspond au féminin. Or l'addition de ces deux nombres primordiaux produit la pentade, appelée aussi « nombre nuptial » ${ }^{38}$, dont Platon souligne la perfection pour ce qui est de favoriser la fécondité matrimoniale ${ }^{39}$, mais qui, selon Plutarque, fournit la clé de la structure du Tout : comme la nature, multiplié par lui-même il reste identique à lui-même ${ }^{40}$. Reprenant les spéculations platoniciennes sur les corps élémentaires et les éléments de la matière ${ }^{41}$, il dérive le premier des nombres pairs de la dyade indéterminée, principe de la pluralité, et le premier des nombres impairs de la monade, principe de l'unité ${ }^{42}$. Nous remontons ainsi à ce qu'il y a de plus essentiel dans l'univers. Au cours de son traité d'herméneutique égyptienne, Isis et Osiris, il applique ces considérations à ces deux divinités, dont le couple symbolise, à ses yeux, l'union du Bien, Osiris, à la Matière, Isis, une union génératrice du monde visible, Horus ${ }^{43}$.

Donc, bien plus qu'à l'accomplissement des rites placés sous le patronage de Déméter ${ }^{44}$, le caractère sacré du mariage tient, d'après Plutarque, au fait qu'il participe au processus continu de l'ordonnance du monde, un processus qu'il reproduit au niveau humain. C'est également en raison de ce caractère que la vie conjugale revêt un aspect éminemment philosophique : sous la conduite du mari, qui, en dépit de l'égalité qualitative reconnue aux femmes, garde chez Plutarque les prérogatives d'un guide protecteur ${ }^{45}$,

37 Page 263 E. Pour l'humidité du corps féminin, voir Quaest. conv. 650 B et 651 C-D.

38 Page 264 A, cf. De E 388 A.

39 R. 546 a-c.

40 Voir De E 388 C-E, cf. 391 C.

41 Voir Ti. $53 \mathrm{c}-56 \mathrm{c}$.

42 Voir De def. or. 427 A - 429 B.

43 Voir le chapitre 56, $373 \mathrm{E}-374 \mathrm{~B}$.

44 Con. praec. $138 \mathrm{~B}$.

45 C'est le rôle dévolu par Plutarque à Pollianos, le jeune marié des Préceptes de mariage (144 D-E ; $144 \mathrm{C}$; 145 B-D). C'est aussi le rôle que Plutarque lui-même joue auprès de 
elle devient une initiation aux mystères de l'ordre du grand Tout.

Il est maintenant possible de conclure. Plutarque se représente le monde sublunaire comme un espace à harmoniser sur le modèle parfait du monde supra-lunaire ${ }^{46}$. Dans cette optique hénologique qui appelle vite l'image de la crase 47 , le mariage fait partie des moyens dont disposent les hommes pour introduire, sous l'inspiration des Muses, c'est-à-dire d'une instance qui les transcende, l'harmonie au sein de la vie politique et sociale. C'est pourquoi les filles de mémoire sont invoquées avec tant d'insistance au début et à la fin des Préceptes de mariage (en $138 \mathrm{C}$ et $146 \mathrm{~A}$ ). Par ses règles l'institution transforme le commerce sexuel dont elle ôte la bestialité en union stable. Plutarque assigne à cette union une fonction très haute, qui dépasse les nécessités de la société, mais dont le principe reste l'amour, un amour que la Muse Érato transmute en amitié fidèle ${ }^{48}$. On ne saurait donc le rapprocher trop ni de Xénophon pour qui le mari doit seulement instruire son épouse sur la manière de gérer un domaine ${ }^{49}$, ni d'Aristote d'après qui le couple demeure naturellement subordonné à l'autorité de l'époux parce que par nature le gouvernant est celui qui garantit la sérénité ${ }^{50}$, ni même de Platon dont les préoccupations apparaissent exclusivement centrées sur la recherche des mesures collectives capables de freiner les processus de corruption inhérents au devenir et qui entraînent la dégénérescence des régimes politiques ${ }^{51}$. Sa position rejoint plutôt l'idéal homérique incarné par le couple d'Alkinoos et Arété ${ }^{52}$; toutefois, il le dépasse, non pas en le couvrant d'un habillage philosophique, mais en lui conférant une dimension presque mystique et en concevant le rôle de l'époux comme celui d'un guide

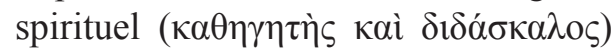
qui ouvre la voie vers les vérités les

sa propre épouse (cf. Cons. ad ux. 611 A et 611 D-F). Auberger (1993) met en évidence que la parité homme-femme -du prétendu modernisme- de Plutarque reste non égalitaire et ne conteste absolument pas le dogme de la supériorité masculine, à laquelle revient de droit le monopole du logos.

46 Voir Quaest. conv. 746 B - 747 A (= livre 9, Problème 14 : À propos du nombre des Muses : traditions minoritaires).

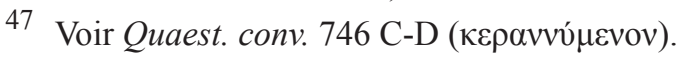

48 Voir Quaest. conv. 746 F.

48 Voir Oec., passim.

50 Voir Pol. 1259 b 1-3 et 1254 b 10-16.

51 Voir R. 546 a - 547 c.

52 Od. VI, 49-78 et 304-315. 


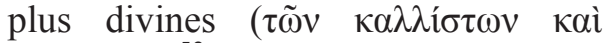
$\theta \varepsilon 10 \tau \alpha \dot{\tau} \tau \omega v)^{53}$ : il ne montre pas la route que doit suivre la femme, à l'instar d'un Iskhomakhos; il aide le couple à se frayer un chemin commun en assumant la responsabilité de la direction.

Une telle conception du mariage sans aucun doute baigne dans l'air d'un temps marqué par la propagation des idées stoïciennes et chrétiennes ${ }^{54}$ et par l'exemple du mariage romain. Mais, à ces influences-là il convient d'en ajouter d'autres, en particulier la revalorisation générale de l'amour au point qu'il devient la thématique principale du roman ${ }^{55}$, où il cesse d'être considéré comme une maladie, transfigure l'existence en lui apportant un sens et trouve son couronnement dans le mariage. Et, bien plus que le mariage romain, ce qui détermine surtout les réflexions de notre auteur sur le sujet se révèle être le mariage d'amour ; il nous le laisse clairement entendre en nous racontant les histoires de Camma et d'Empona, respectivement une Galate et une Gauloise, dont la tragédie offre deux exemples paradigmatiques de fidélité conjugale parfaite ${ }^{56}$.

Pour toutes ces raisons, il vaudrait mieux parler d'une véritable vocation $\mathrm{du}$ mariage chez Plutarque, une vocation qu'il théorise, au fil de son œuvre, à partir de son expérience personnelle du mariage d'amour ${ }^{57}$ qu'il a vécu avec Timoxéna.

\section{Bibliographie}

\section{Auteurs anciens}

Alexander Aphrodisiensis,

- De Mixtione.

Aristoteles,

- De generatione et corruptione.

- Politica

Hesiodus,

- Opera et dies.

- Theogonia.

Hippocrates,

- De natura hominis.

Homerus,

- Ilias.

- Odyssea.

Philo Judaeus,

- De confusione linguarum.

Plato,

- Lysis.

- Symposium.

- Timaeus.

- Respublica 8.

Plutarchus,

- Conjugalia praecepta.

- Mulierum virtutes.

- Amatorius.

- Quaestiones Romanae.

53 Con. praec. 145 C.

54 Voir, là-dessus, Foucault, (1984), 177-192 (« Le lien conjugal »); 193-205 (« La question du monopole ») ; 206-216 (« Les plaisirs du mariage »).

55 Voir Fusillo (1991), 195-257.

56 Voir Amatorius 768 B-D et 770 D - 771 C.

57 Voir Amatorius 749 B et 771 D-E. 
- De tuenda sanitate praecepta.

- Quaestiones convivales.

- De communibus notitiis adversus Stoicos.

- De defectu oraculorum.

- Septem sapientium convivium.

- De E apud Delphos.

- Consolatio ad uxorem.

Seneca,

- De beneficiis.

Xenopho,

- Oeconomicus.

\section{Éditions}

BABBITT, F.C.,

- Plutarch's Moralia, Vol. II, LondonCambridge Mass.(LCL), 1971.

Defradas, J. \& Klaerr, R.,

- Plutarque. Euvres Morales, Tome II, Paris (CUF), 1985.

JOUANNA, J.,

- Hippocrate. La nature de l'homme, Berlin (CMG I, 1, 3), 1975.

Martano, G. \& Tirelli, A.,

- Plutarco. Precetti conjugali, Napoli (Corpus Plutarchi Moralium, 6), 1990.

SANDBACH, F.H.,

- Plutarch's Moralia, Vol. XV, Fragments, London-Cambridge Mass. (LCL), 1969.

TodD, R.B.,

- Alexander Aphrodisiensis. A study of the "De mixtione", Leiden, 1976.

Von Arnim, J.,

- Stoicorum Veterum Fragmenta, II, editio stereotypa (1964), Stuttgart (BGT), 1905.

\section{Monographies}

BABut, D.,

- Plutarque et le stö̈cisme, Paris, 1969.

Balzac, H.,

- Physiologie du mariage, ou méditation de philosophie éclectique sur le bonheur et le malheur conjugal, réédité in : Castex P.-J. (éditeur) (1980), Balzac. La Comédie humaine, Volume 11, Paris, 1829, 865-1205.

BENVÉNISTE, É.,

- Le vocabulaire des institutions indoeuropéennes, volume 1, Paris, 1969.

Foucault, M.,

- Le souci de soi, Paris, 1984.

Fusillo, M.,

- Il Romanzo greco. Polifonia ed Eros, Venezia, traduction française in : Abrioux $\mathrm{M}$. (1991), Naissance du roman, Paris, 1989.

KAHN, L.,

- Hermès passe, ou les ambiguïtés de la communication, Paris, 1978.

\section{Articles}

Auberger, J.,

- «Parole et silence dans les Préceptes de mariage de Plutarque », in : LEC, 61 (1993) 297-308.

Nikolaidis, A.G.,

- "Plutarch on Women and Marriage », in : Wiener Studien, 110 (1997) 27-88.

PatTerson, C.,

- «Plutarch's Advices on Marriage ; Traditional Wisdom through a Philosophic Lens ", in: $A N R W 2.36 .6,1992,4709-4723$. 